\title{
Electric field stimulation setup for photoemission electron microscopes
}

\author{
M. Buzzi, C. A. F. Vaz, J. Raabe, and F. Nolting ${ }^{\text {a) }}$ \\ Paul Scherrer Institut, 5232 Villigen PSI, Switzerland
}

(Received 26 March 2015; accepted 23 July 2015; published online 6 August 2015)

\begin{abstract}
Manipulating magnetisation by the application of an electric field in magnetoelectric multiferroics represents a timely issue due to the potential applications in low power electronics and the novel physics involved. Thanks to its element sensitivity and high spatial resolution, X-ray photoemission electron microscopy is a uniquely suited technique for the investigation of magnetoelectric coupling in multiferroic materials. In this work, we present a setup that allows for the application of in situ electric and magnetic fields while the sample is analysed in the microscope. As an example of the performances of the setup, we present measurements on $\mathrm{Ni} / \mathrm{Pb}\left(\mathrm{Mg}_{0.66} \mathrm{Nb}_{0.33}\right) \mathrm{O}_{3^{-}}$ $\mathrm{PbTiO}_{3}$ and $\mathrm{La}_{0.7} \mathrm{Sr}_{0.3} \mathrm{MnO}_{3} / \mathrm{PMN}-\mathrm{PT}$ artificial multiferroic nanostructures. (C) 2015 AIP Publishing LLC. [http://dx.doi.org/10.1063/1.4927825]
\end{abstract}

\section{INTRODUCTION}

Magnetoelectric multiferroics exhibit the coexistence of two primary ferroic orders: ferromagnetism and ferroelectricity. Thanks to the presence of a magnetoelectric coupling in these materials, it is possible to manipulate the magnetic properties by the sole application of an electric field, with low power dissipation. ${ }^{1}$ This last ability is of great interest for the development of new devices that meet the requirements for high speed and low energy consumption. While it is difficult to fulfil device-suitable operating conditions using single phase multiferroics, it is possible to overcome this problem by coupling two different ferroic materials to induce a magnetoelectric coupling at the interface. ${ }^{2-6}$ In this context, magnetisation reorientations ${ }^{7,8}$ and displacement of magnetic domain walls ${ }^{9,10}$ with the application of electric fields have been demonstrated at ambient temperature.

Traditionally, magnetoelectric coupling is investigated by spatially averaging techniques such as dielectric measurements, ${ }^{11}$ magneto-transport measurements, ${ }^{7}$ superconducting quantum interference device magnetometry, ${ }^{12,13}$ and magnetooptic Kerr effect. ${ }^{14,15}$ Recently, the use of spatially resolved techniques capable of imaging single ferroic domains was proven to be crucial to achieve a more detailed understanding of the mechanisms at the origin of magnetoelectric coupling in complex multiferroic systems. ${ }^{16,17}$ Scanning probe techniques such as magnetic force microscopy and piezoresponse force microscopy are nowadays employed in the study of multiferroics materials. However, they have intrinsic shortcomings, especially when employed in the investigation of artificial multiferroics. Piezoresponse force microscopy is reliable only for ferroelectric layers having an exposed, uncoated surface. ${ }^{18}$ However, due to growth constraints, especially in the case of epitaxial artificial multiferroic heterostructures, it is not always possible to arrange the layer stack to meet this requirement. Second, contrast in magnetic force microscopy can be affected by the presence of modulations in the

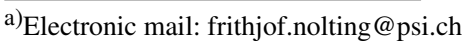

surface potential. The presence of adjacent ferroelectric domains with polarisation pointing in different directions can cause such modulations and give rise to artefacts in the magnetic image.

X-ray photoemission electron microscopy (X-PEEM) allows for simultaneous imaging of ferroelectric and ferromagnetic domains down to $50 \mathrm{~nm}$ spatial resolution. In this case, the contrast mechanism for ferroic domains relies on the $\mathrm{x}$ ray magnetic circular dichroism (XMCD) effect for ferromagnetic domains ${ }^{19}$ and $\mathrm{x}$-ray linear dichroism (XLD) effect for ferroelectric domains. ${ }^{20,21}$ By purposely tuning the incoming photon energy to the appropriate absorption edge and selecting the correct $\mathrm{x}$-ray polarisation, one can investigate ferroelectric and ferromagnetic domains independently. Moreover, despite being well known for its surface sensitivity, X-PEEM has probing depths of $5 \mathrm{~nm}$ and $10 \mathrm{~nm}$ that allow for the investigation of buried layers.

Recently, the capabilities of X-PEEM have been extended in order to transform it from an instrument mainly oriented to solve surface science problems to a tool that takes full advantage of synchrotron radiation to investigate the effect of in situ stimuli in complex materials. Several reports focus on the instrumentation for applying magnetic fields, current pulses, and ultrashort laser pulses during X-PEEM imaging, ${ }^{22-27}$ in order to study the response of ferromagnetic materials to these excitations. In this work, we report a novel development in X-PEEM that allows for the application of in situ electric field stimuli during imaging. This new opportunity further extends the capabilities of X-PEEM to the investigation of magnetoelectric coupling in multiferroics and, more generally, to the study of electrostatic field effect phenomena at functional interfaces. Our setup allows for the application of voltages as high as $1 \mathrm{kV}$, enabling the investigation of a wide variety of multiferroic systems. It is designed to be integrated with the ELMITEC PEEM microscope, which is available in multiple synchrotron light sources as a user facility, but thanks to its modularity, our setup can be easily adapted to different photoemission microscopes. At last, we illustrate the capabilities of our experimental device by investigating an electric field-induced magnetisation 
reorientation in $\mathrm{Ni} / \mathrm{Pb}\left(\mathrm{Mg}_{0.33} \mathrm{Nb}_{0.66}\right) \mathrm{O}_{3}-\mathrm{PbTiO}_{3}$ (PMN-PT) and $\mathrm{La}_{0.7} \mathrm{Sr}_{0.3} \mathrm{MnO}_{3}$ (LSMO)/PMN-PT artificial multiferroic nanostructures.

\section{INSTRUMENTATION DESIGN}

\section{A. Intrinsic requirements}

During X-PEEM imaging, the sample under investigation is illuminated by $\mathrm{x}$-ray photons. Upon absorption of the incoming photons, electrons with a kinetic energy greater than the work function of the sample are photoemitted and collected by the objective lens of the microscope. A full-field magnified image of the illuminated area is then formed on the detector by means of a set of electron lenses. In order to make this possible, the photo-emitted electrons are accelerated by a constant acceleration voltage of typically $-10 \mathrm{kV}$ to $-20 \mathrm{kV}$ which is applied between the objective lens of the microscope and the sample. $^{28}$

In the ELMITEC PEEM microscope, the objective lens is connected to earth ground and the accelerating voltage is directly applied to the sample. An additional bias identified as start voltage is also applied in order to define the centre energy of the photo-emitted electrons accepted by the energy analyser. As a result, the sample ground is biased with respect to earth ground by both the accelerating and the start voltages (Fig. 1). All equipment that for experimental reasons are electrically connected to the sample has to be referenced to the sample ground. To ease this task, the microscope control rack is equipped with an isolated 19 in. rack identified as high voltage rack which is biased to the accelerating voltage and that houses the start voltage power supply as well as the

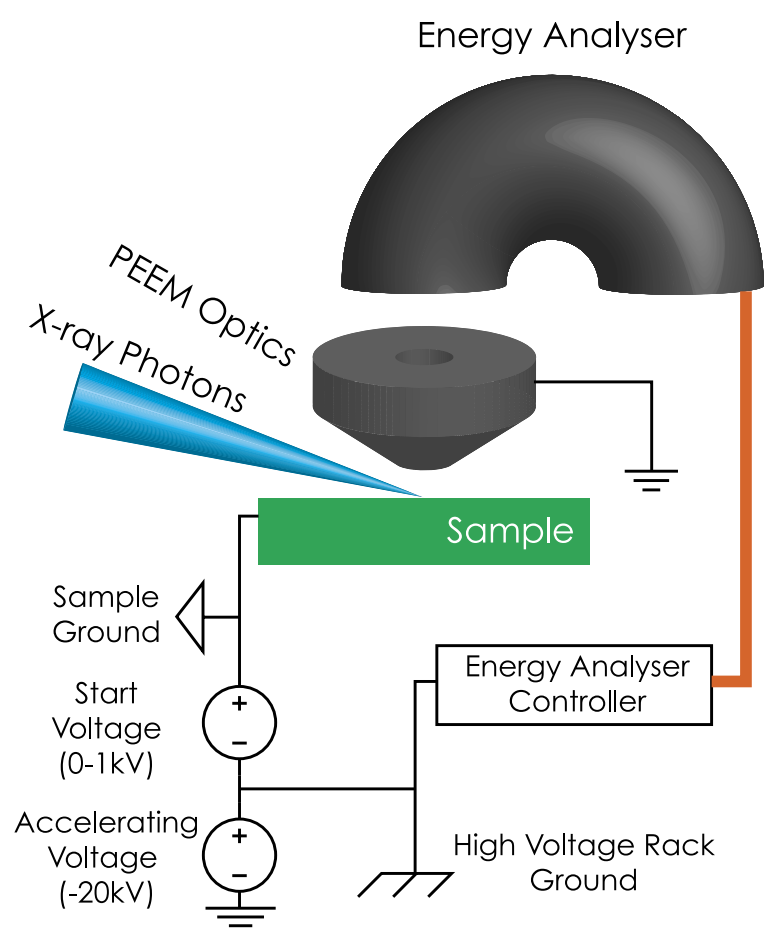

FIG. 1. Simplified schematic of the ELMITEC PEEM microscope, highlighting the bias voltages necessary for the operation of the microscope. sample heating, LEEM, and energy analyser controllers. The six electrical connections to the sample are terminated in this rack.

When a new instrument is integrated in the high voltage rack, two main aspects have to be considered. (i) The exchange of data for instrument control and/or data acquisition purposes has to happen via optical links to maintain the required electrical isolation. (ii) Due to the high electric field $\left(\approx 10 \mathrm{kV} \mathrm{mm}^{-1}\right)$ present between the sample and the PEEM objective lens, arcing may occur, creating a risk of damage for all the instrumentation electrically connected to the sample if appropriate safety measures are not taken. To fulfil these requirements, one needs to develop fully customised solutions or to carefully integrate commercial instruments with adequate protection.

To achieve our goal of applying in situ electric field stimuli during PEEM measurements, we have introduced the following modifications to the ELMITEC PEEM microscope.

- A sample holder that allows for electrical connections to the sample.

- A computer controlled unit that can generate analog control voltages for driving commercial instruments.

- Integrated a commercial high voltage amplifier in the high voltage rack with adequate protection.

\section{B. Sample mounting and sample holder}

When studying how the magnetic domain configuration in magnetoelectric multiferroics is affected by the application of an electric field, it is important to take particular care in avoiding the presence of stray electric fields at the sample surface that may cause image aberrations. The standard ELMITEC sample holder clamps the sample against the metallic body of the sample holder using a round clip. While this type of sample holder is optimised for surface science experiments involving sample preparations at high temperature, it is not suitable for the application of electric and magnetic fields. We adopted a stainless steel sample holder with four electrical contacts as shown in Figure 2(a). All of the contacts can be used to provide a constant bias voltage to the sample in order to generate an in situ electric field. The sample holder also allows for the installation of a small electromagnet that can generate magnetic fields up to $15 \mathrm{mT}^{24}$ When the electromagnet is installed, only two contacts are available to apply bias voltages to the sample.

Artificial multiferroic samples based on single crystal ferroelectrics such as schematically shown in Figure 2(b) are mounted directly on the top cover of the sample holder using four small drops of silver glue. This mounting technique ensures that the sample is held to the top cover with minimum residual stress and, at the same time, that the top electrode is connected to the sample ground. A Kapton ${ }^{\mathrm{TM}}$ insulated copper wire is then glued using silver epoxy to the back electrode and then connected to one of the free electrical contacts on the sample holder. In this way, the electric field across the sample is generated by applying a bias potential on the back electrode while keeping the top electrode at sample ground in order to avoid spurious electric fields that may cause image distortions. The top cover is fixed to the main body of the sample holder 

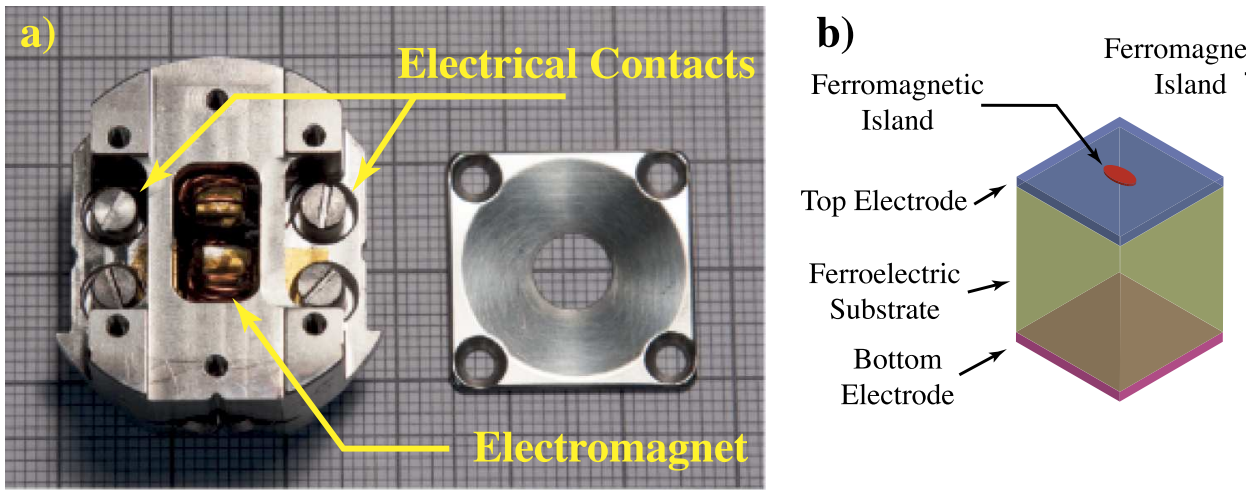

c)
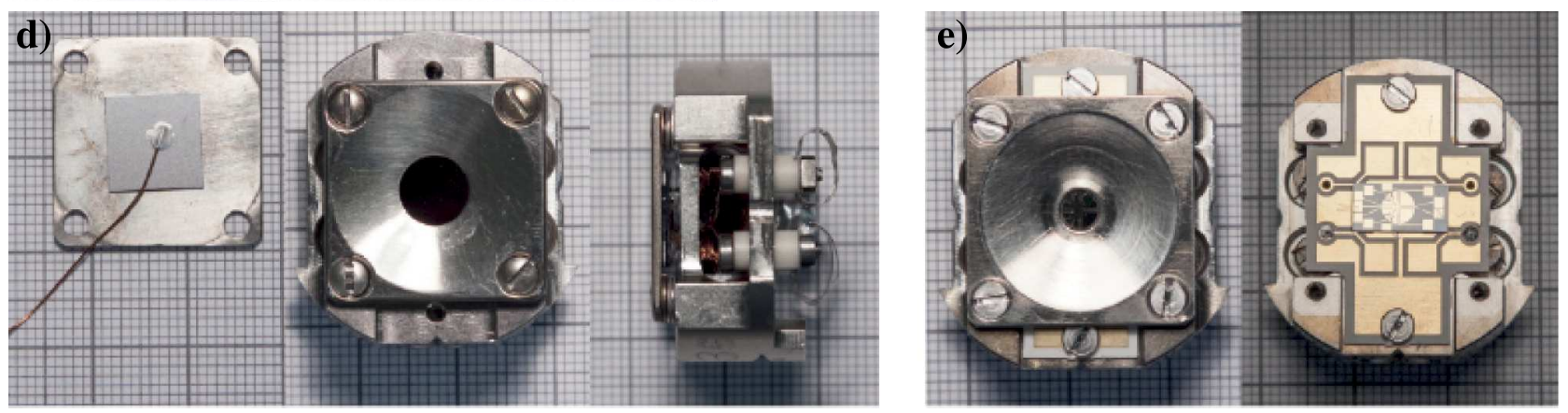

FIG. 2. (a) Overall view of the sample holder used for PEEM measurements with in situ applied electric and magnetic fields. (b) Typical structure for artificial multiferroic samples based on single crystal piezoelectrics. (c) Typical structure for thin film artificial multiferroics. Both the ferromagnetic and ferroelectric constituents are in the form of thin films. (d) Mounting procedure for artificial multiferroic samples based on single crystal piezoelectrics. (e) Mounting procedure for thin film artificial multiferroic heterostructures. All the pictures are taken using millimeter paper as a background.

using four screws. A distance of $\approx 1 \mathrm{~mm}$ between the sample back electrode and the top part of the sample holder is kept by means of a combination of four spacers and four spring washers that allow to null the sample tilt prior to transferring the sample in vacuum. A side and top view of the fully assembled sample holder is shown in Figure 2(d).

In the case of thin film artificial multiferroics, the ferroelectric and ferromagnetic layers are both in the form of thin layers deposited on an insulating substrate as shown in Figure 2(c). The connections to the electrodes for applying an electric field to the ferroelectric layer are achieved either via wire-bonding or by gluing thin gold wires with silver glue to the connection pads defined lithographically on the sample. The connecting wires are then terminated on a circuit board carrier (Fig. 2(e)) that is installed directly on the sample holder. The circuit board carrier is manufactured using a PTFE based laminate (Rogers RO-3003) characterised by a low outgassing rate under ultra high vacuum. The sample carrier board can then be connected to one of the free contacts of the sample holder. The top cover is put in place using spacers to maintain a gap of $\leq 0.3 \mathrm{~mm}$ with respect to the sample surface. It is important to make sure that the top cover shadows all the bonding wires to the sample to avoid field emission effects that would lead to the destruction of the sample. Figure 2(e) shows a sample mounted using this method.

\section{Control Unit}

The reference ground for all the instruments electrically connected to the sample is the sample ground. It differs from the high voltage rack ground by the start voltage as shown in
Figure 1. As most commercial instruments are not designed to withstand a voltage difference between their chassis ground and the input or output terminals of more than few hundreds volts, we have prepared an isolated $19 \mathrm{in}$. shelf that can be installed in the high voltage rack and connected to sample ground. In order to maintain the required electrical isolation, the instruments installed in this isolated shelf are powered by a 100 VA toroidal isolation transformer.

Figure 3 shows a block diagram of the setup. All the components are controlled by a home built control unit based on an Atmel ATmega 2560 micro-controller. All the required functions can be controlled by sending SCPI commands via a serial link on optical fibre. To generate the voltage required to apply electric fields at the sample position, the unit sets $\mathrm{a} \pm 10 \mathrm{~V}$ analog output which is connected to a bipolar high voltage amplifier (Trek 2210) that can supply up to $\pm 1 \mathrm{kV}$. A small relay box built using high voltage reed relays routes the output of the high voltage amplifier to the correct contact on the sample holder.

To prevent damage of the output stage of the high voltage amplifier in the event of a discharge between the sample and the PEEM objective lens, we designed a surge protection circuit. We found that the best solution for a reliable protection circuit is to connect a combination of a $1 \mathrm{kV}$ transient suppressor diode and a gas discharge tube to each output terminal of the high voltage amplifier. Figure 4 shows a detailed schematic of how the surge protection circuit is implemented.

The electromagnetic coil installed in the sample holder is driven using the constant current source integrated in the ELMITEC sample heater controller and a low voltage relay box allows for reversing the polarity of the magnetic field or 


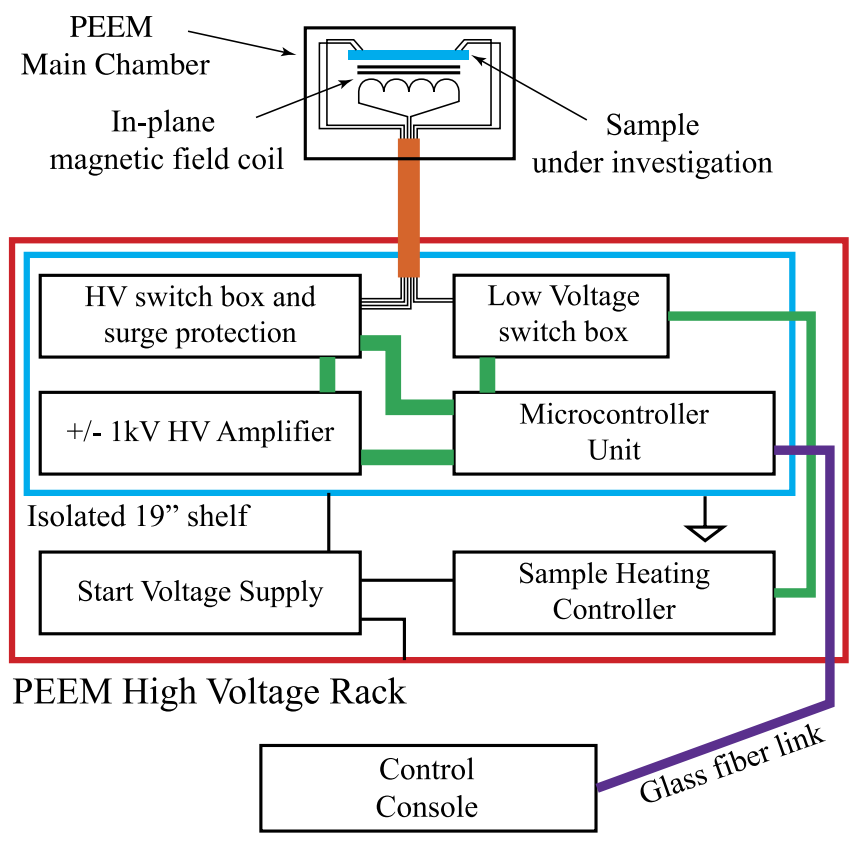

FIG. 3. Block diagram of the setup. The energy analyser and electron gun controller which are normally present in the microscope high voltage rack are not shown for sake of simplicity. A fiber-optic link ensures the communication between the microcontroller and an external computer.

through a home-built bipolar, \pm 3 A current amplifier. Typically, during an experiment, the magnetic state of the sample is initialised using the electromagnet and then the electric field is swept in steps while acquiring images at each step to study how the application of the electric field affects the magnetic domain configuration.

\section{PROOF-OF-PRINCIPLE MEASUREMENTS}

To demonstrate the capabilities of the present setup, we report on X-PEEM measurements in applied electric fields of the ferromagnetic domain configuration of lithographically defined $\mathrm{Ni}$ and $\mathrm{La}_{0.7} \mathrm{Sr}_{0.3} \mathrm{MnO}_{3}$ nanostructures deposited on PMN-PT (011) ferroelectric single crystals.

To image the ferromagnetic domain configuration of the samples, we employ the XMCD effect by tuning the incoming photon energy to the Ni- $L_{3}$ edge $(\approx 852 \mathrm{eV})$ and $\mathrm{Mn}-L_{3}$ edge

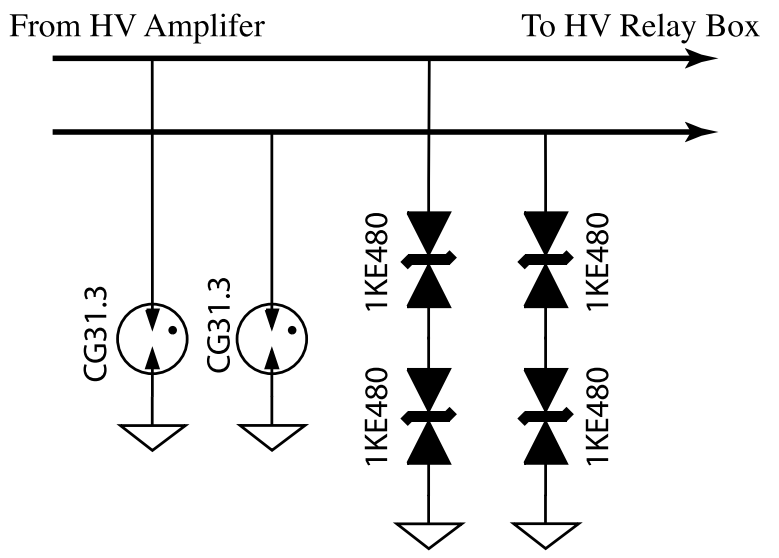

FIG. 4. Schematic diagram for the surge protection circuit integrated in the high voltage switch box. a)

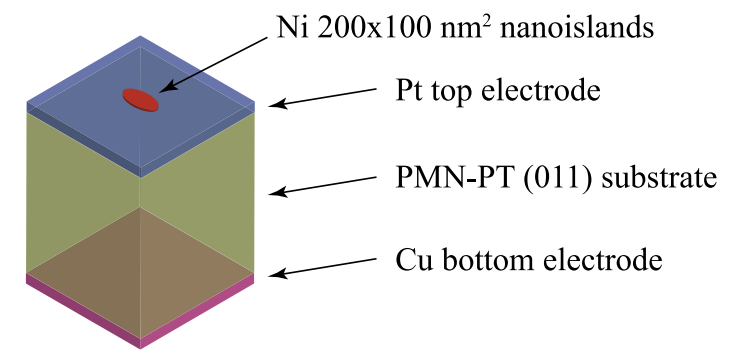

b)
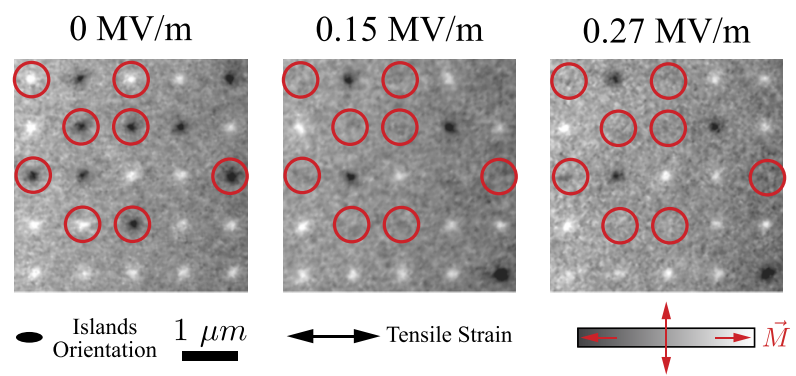

FIG. 5. (a) Sketch of the Ni/PMN-PT sample structure. (b) Sequence of successive XMCD images acquired at the $\mathrm{Ni}-L_{3}$ edge for different applied electric fields without applied magnetic field. Islands showing a $90^{\circ}$ magnetisation reorientation are highlighted with red circles. Each XMCD image is the result of the averaging of 10 single XMCD images acquired with a total exposure time of $8 \mathrm{~s}$. Reproduced with permission from Buzzi et al., Phys. Rev. Lett. 111, 027204 (2013). Copyright (2013) by the American Physical Society.

$(\approx 641 \mathrm{eV})$ and acquiring images with opposite light helicities $\left(c_{+}\right.$and $\left.c_{-}\right)$. By calculating the asymmetry $\left(c_{+}-c_{-}\right) /\left(c_{+}+c_{-}\right)$, one isolates the magnetic contrast, which is proportional to $\vec{M}(\vec{r}) \cdot \vec{k}$, where $\vec{k}$ is the propagation vector of the incoming X-rays. ${ }^{27}$ For clarity, the XMCD image shows black or white contrast in areas where magnetic moments have a component parallel or antiparallel to $\vec{k}$. The experiments presented here were carried out at the Surface/Interface Microscopy (SIM) beamline of the Swiss Light Source. ${ }^{29}$

Figure 5(a) shows a sketch of the Ni/PMN-PT sample structure; details about the sample fabrication are reported elsewhere. ${ }^{17}$ The electric field is applied to the ferroelectric by applying a bias voltage between the $\mathrm{Pt}$ and the $\mathrm{Cu}$ electrodes. Figure 5(b) shows a series of XMCD images of a $5 \times 5$ array of $200 \times 100 \mathrm{~nm}^{2} \mathrm{Ni}$ islands having their long axis parallel to the $[01 \overline{1}]$ direction of the PMN-PT crystal. Prior to imaging, we initialised the magnetic and ferroelectric configurations of the sample following an $\mathrm{AC}$ demagnetisation protocol and by poling the ferroelectric with an electric field of $-0.4 \mathrm{MV} \mathrm{m}^{-1}$. The XMCD series was then acquired with no applied magnetic field and sweeping the electric field in steps from $0 \mathrm{MV} \mathrm{m}^{-1}$ to $0.27 \mathrm{MV} \mathrm{m}^{-1}$. We observe that in the initial configuration at $0 \mathrm{MV} \mathrm{m}^{-1}$, all the islands show a uniform contrast indicating that they are uniformly magnetised in a direction parallel (black) or anti-parallel (white) to x-ray propagation direction. No changes are observed until the electric field is increased up to the ferroelectric coercive field $E \simeq E_{c}=0.15 \mathrm{MV} \mathrm{m}^{-1}$. At this point, the contrast of the eight islands highlighted with red circles changes from white or black to grey showing that the magnetisation is now aligned perpendicular to the $\mathrm{x}$-ray propagation direction. No further changes are observed for larger electric fields. 
Here, we focus only on the highlighted islands. A more complete model that accounts for the behaviour of all the islands is published elsewhere. ${ }^{17}$ The observed $90^{\circ}$ magnetisation reorientation can be explained in the scenario of strain-mediated magnetoelectric coupling. At the ferroelectric coercive field, $E_{c}$, PMN-PT induces a strong tensile strain along the $[01 \overline{1}]$ direction and a smaller compressive strain along the orthogonal [100] direction. The strain applied to the polycrystalline $\mathrm{Ni}$ islands is responsible for an additional magnetoelastic anisotropy term. It can be modelled using a uniaxial magnetic anisotropy term as $U_{m e}=-(3 / 2) \lambda_{p} Y\left(\varepsilon_{01 \overline{1}}-\varepsilon_{100}\right)$ $\cos ^{2} \theta_{01 \overline{1}}$, where $\lambda_{p}=-3.4 \times 10^{-5}$ is the magnetostriction coefficient of polycrystalline Ni, $Y=220 \mathrm{GPa}$ is the Ni Young's modulus, and $\left(\varepsilon_{01 \overline{1}}-\varepsilon_{100}\right)$ is the strain applied to the $\mathrm{Ni}$ by the PMN-PT. For islands with their long axis parallel to the [011] direction of the PMN-PT, positive values of $\left(\varepsilon_{01 \overline{1}}-\varepsilon_{100}\right)$ generate a positive magnetoelastic contribution that acts against the magnetostatic term (shape anisotropy) and favours a reorientation of the magnetisation to the short axis of the island. The magnetostatic energy barrier to overcome to keep the magnetisation parallel to the island short axis can be estimated using an analytical model for ferromagnetic prisms ${ }^{30}$ and is approximately $10 \mathrm{~kJ} \mathrm{~m}^{-3}$. At the ferroelectric coercive field, $\left(\varepsilon_{01 \overline{1}}-\varepsilon_{100}\right)$ is approximately $1300 \mathrm{ppm}$ and the magnetoelastic contribution is $\approx 15 \mathrm{~kJ} \mathrm{~m}^{-3}$ making the long axis of the islands a hard magnetisation direction and inducing a $90^{\circ}$ reorientation to the island short axis.

Figure 6(a) shows a sketch of a patterned LSMO/PMNPT epitaxial heterostructure; details about the growth and patterning of the LSMO layer are reported elsewhere. ${ }^{31,32}$ The thickness of the LSMO layer is $17 \mathrm{~nm}$ and the electric field is applied to the PMN-PT by applying a bias voltage between the $\mathrm{Cu}$ back electrode and the top LSMO amorphous matrix that has a low enough resistance to effectively allow one to apply a static electric field across the ferroelectric. Figure 6(b) shows a series of XMCD images for different applied electric fields of an epitaxial LSMO $45^{\circ}$ zig-zag wire of $1 \mu \mathrm{m}$ width. The wire extends $45^{\circ}$ off the [100] direction meaning that each of

a)

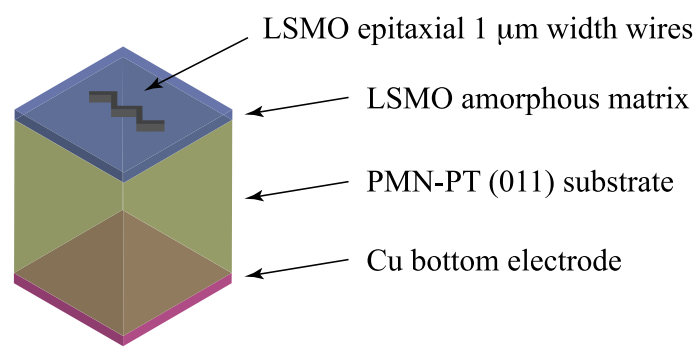

b)

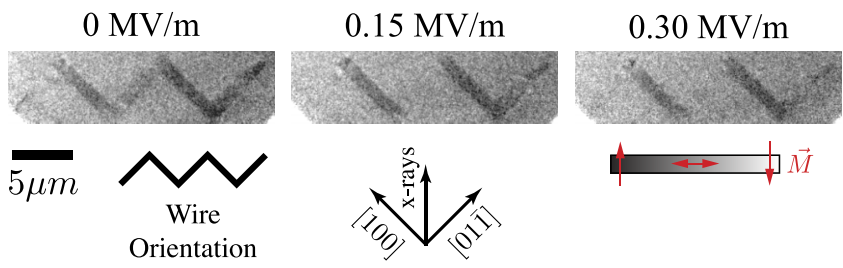

FIG. 6. (a) Sketch of the sample structure. (b) Sequence of successive XMCD images of a $1 \mu \mathrm{m}$ width $45^{\circ}$ zig-zag wire acquired at the Mn- $L_{3}$ edge for different electric fields and without applied magnetic field. its segments extends alternatively along the [100] or the [011] direction as indicated in the sketch in Figure 6(b). The XMCD sequence was recorded with no applied magnetic field after poling the ferroelectric at $-0.5 \mathrm{MV} \mathrm{m}^{-1}$ and applying a $15 \mathrm{mT}$ magnetic field pulse along the $\mathrm{x}$-ray propagation direction. Analysing the first XMCD image acquired at $0 \mathrm{MV} \mathrm{m}^{-1}$, we observe that both the segments along the [100] and [011] directions show a uniform dark grey contrast indicating that they are uniformly magnetised and suggesting that in this state, the magnetic anisotropy is fourfold. When the electric field is increased to the ferroelectric coercive field $E_{c}=0.15 \mathrm{MV} \mathrm{m}^{-1}$, we observe that the contrast for the segments oriented along the [011] direction decreases suggesting that the [011] direction is now a less favourable direction for the magnetisation and the magnetic anisotropy in this configuration is twofold. No further changes are observed for larger electric fields.

\section{CONCLUSIONS}

In conclusion, we have presented an experimental device for X-PEEM that enables the stimulation of the sample under investigation with both electric and magnetic fields. We have demonstrated the capabilities of the setup by investigating magnetoelectric coupling in Ni/PMN-PT and LSMO/PMNPT heterostructures. The observed behaviour reveals the presence of a strain induced magnetoelectric coupling which is at the origin of the electric field assisted magnetisation reorientations we have observed.

Finally, the flexibility of our setup enables the use of XPEEM for the investigation of electric field-induced effects in a large variety of complex oxides and functional interfaces such as bi-dimensional electron gas systems, insulating antiferromagnets, topological insulators, and magnetic molecules.

\section{ACKNOWLEDGMENTS}

The authors would like to thank Mathias Kläui for fruitful discussions, R. V. Chopdekar and J. L. Hockel for providing the samples used during the test measurements, and Pascal Schifferle and Juri Honegger for technical support. Part of this work was performed at the Swiss Light Source, Paul Scherrer Institute, Villigen, Switzerland. This work was partially funded by EUs 7th Framework Program IFOX (No. NMP3LA-2010 246102).

\footnotetext{
${ }^{1}$ W. Eerenstein, N. D. Mathur, and J. F. Scott, Nature 442, 759 (2006).

${ }^{2}$ N. A. Spaldin and M. Fiebig, Science 309, 391 (2005).

${ }^{3}$ M. Fiebig, J. Phys. D: Appl. Phys. 38, R123 (2005).

${ }^{4}$ C. A. F. Vaz, J. Phys.: Condens. Matter 24, 333201 (2012).

${ }^{5}$ C. A. F. Vaz and U. Staub, J. Mater. Chem. C 1, 6731 (2013).

${ }^{6}$ V. Garcia, M. Bibes, and A. Barthélémy, C. R. Phys. 16, 168 (2015).

${ }^{7}$ J. T. Heron, M. Trassin, K. Ashraf, M. Gajek, Q. He, S. Y. Yang, D. E. Nikonov, Y.-H. Chu, S. Salahuddin, and R. Ramesh, Phys. Rev. Lett. 107, $217202(2011)$.

${ }^{8}$ J. T. Heron, J. L. Bosse, Q. He, Y. Gao, M. Trassin, L. Ye, J. D. Clarkson, C. Wang, J. Liu, S. Salahuddin, D. C. Ralph, D. G. Schlom, J. Iniguez, B. D. Huey, and R. Ramesh, Nature 516, 370 (2014).

${ }^{9}$ J. L. Hockel, A. Bur, T. Wu, K. P. Wetzlar, and G. P. Carman, Appl. Phys. Lett. 100, 022401 (2012).

${ }^{10}$ S. Finizio, M. Foerster, M. Buzzi, B. Krüger, M. Jourdan, C. A. F. Vaz, J. Hockel, T. Miyawaki, A. Tkach, S. Valencia, F. Kronast, G. P. Carman, F. Nolting, and M. Kläui, Phys. Rev. Appl. 1, 021001 (2014).
} 
${ }^{11}$ T. Kimura, Y. Sekio, H. Nakamura, T. Siegrist, and A. P. Ramirez, Nat. Mater. 7, 291 (2008).

${ }^{12}$ P. M. Leufke, R. Kruk, R. A. Brand, and H. Hahn, Phys. Rev. B 87, 094416 (2013).

${ }^{13}$ P. Borisov, A. Hochstrat, V. V. Shvartsman, and W. Kleemann, Rev. Sci. Instrum. 78, 106105 (2007).

${ }^{14}$ C. A. F. Vaz, J. Hoffman, Y. Segal, J. W. Reiner, R. D. Grober, Z. Zhang, C. H. Ahn, and F. J. Walker, Phys. Rev. Lett. 104, 127202 (2010).

${ }^{15}$ T. Wu, A. Bur, P. Zhao, K. P. Mohanchandra, K. Wong, K. L. Wang, C. S. Lynch, and G. P. Carman, Appl. Phys. Lett. 98, 012504 (2011).

${ }^{16}$ S. Zhang, Y. G. Zhao, P. S. Li, J. J. Yang, S. Rizwan, J. X. Zhang, J. Seidel, T. L. Qu, Y. J. Yang, Z. L. Luo, Q. He, T. Zou, Q. P. Chen, J. W. Wang, L. F. Yang, Y. Sun, Y. Z. Wu, X. Xiao, X. F. Jin, J. Huang, C. Gao, X. F. Han, and R. Ramesh, Phys. Rev. Lett. 108, 137203 (2012).

${ }^{17}$ M. Buzzi, R. V. Chopdekar, J. L. Hockel, A. Bur, T. Wu, N. Pilet, P. Warnicke, G. P. Carman, L. J. Heyderman, and F. Nolting, Phys. Rev. Lett. 111, 027204 (2013).

${ }^{18}$ E. Soergel, J. Phys. D: Appl. Phys. 44, 464003 (2011).

${ }^{19}$ J. Stohr, Y. Wu, B. D. Hermsmeier, M. G. Samant, G. R. Harp, S. Koranda, D. Dunham, and B. P. Tonner, Science 259, 658 (1993).

${ }^{20}$ R. V. Chopdekar, V. K. Malik, A. Fraile Rodriguez, L. Le Guyader, Y. Takamura, A. Scholl, D. Stender, C. W. Schneider, C. Bernhard, F. Nolting, and L. J. Heyderman, Phys. Rev. B 86, 014408 (2012).

${ }^{21}$ T. Zhao, A. Scholl, F. Zavaliche, K. Lee, M. Barry, A. Doran, M. P. Cruz, Y. H. Chu, C. Ederer, N. A. Spaldin, R. R. Das, D. M. Kim, S. H. Baek, C. B. Eom, and R. Ramesh, Nat. Mater. 5, 823 (2006).
${ }^{22}$ T. Kinoshita, T. Ohkochi, H. Osawa, K. Arai, K. Fukumoto, T. Okuda, M. Kotsugi, T. Muro, T. Nakamura, and T. Matsushita, J. Electron Spectrosc. Relat. Phenom. 185, 389 (2012).

${ }^{23}$ O. Sandig, J. Herrero-Albillos, F. M. Römer, N. Friedenberger, J. Kurde, T. Noll, M. Farle, and F. Kronast, J. Electron Spectrosc. Relat. Phenom. 185, 365 (2012).

${ }^{24}$ L. Heyne, M. Kläui, J. Rhensius, L. Le Guyader, and F. Nolting, Rev. Sci. Instrum. 81, 113707 (2010).

${ }^{25}$ J. Miguel, M. Bernien, D. Bayer, J. Sánchez-Barriga, F. Kronast, M. Aeschlimann, H. A. Dürr, and W. Kuch, Rev. Sci. Instrum. 79, 033702 (2008).

${ }^{26}$ L. Gierster, L. Pape, A. A. Ünal, and F. Kronast, Rev. Sci. Instrum. 86, 023702 (2015).

${ }^{27}$ L. Le Guyader, A. Kleibert, A. F. Rodriguez, S. E. Moussaoui, A. Balan, M. Buzzi, J. Raabe, and F. Nolting, J. Electron Spectrosc. Relat. Phenom. 185, 371 (2012).

${ }^{28}$ E. Bauer, J. Phys.: Condens. Matter 13, 11391 (2001).

${ }^{29}$ U. Flechsig, F. Nolting, A. F. Rodriguez, J. Krempasky, C. Quitmann, T. Schmidt, S. Spielmann, and D. Zimoch, AIP Conf. Proc. 1234, 319 (2010).

${ }^{30}$ A. Aharoni, J. Appl. Phys. 83, 3432 (1998).

${ }^{31}$ Y. Takamura, R. V. Chopdekar, A. Scholl, A. Doran, J. A. Liddle, B. Harteneck, and Y. Suzuki, Nano Lett. 6, 1287 (2006).

${ }^{32}$ J. Heidler, C. Piamonteze, R. V. Chopdekar, M. A. Uribe-Laverde, A. Alberca, M. Buzzi, A. Uldry, B. Delley, C. Bernhard, and F. Nolting, Phys. Rev. B 91, 024406 (2015). 